\title{
Free vibration analysis of rectangular plate with arbitrary edge constraints using characteristic orthogonal polynomials in assumed mode method
}

\author{
Kookhyun Kim ${ }^{1}$, Byung-Hee Kim ${ }^{2}$, Tae-Muk $\mathrm{Choi}^{3}$ and Dae-Seung Cho ${ }^{4}$ \\ ${ }^{I}$ Department of Naval Architecture, Tongmyong University, Busan, Korea \\ ${ }^{2}$ Marine Research Institute, Samsung Heavy Industries Co. Ltd., Geoje, Korea \\ ${ }^{3}$ Createch Co. Ltd., Busan, Korea \\ ${ }^{4}$ Department of Naval Architecture and Ocean Engineering, Pusan National University, Busan, Korea
}

\begin{abstract}
An approximate method based on an assumed mode method has been presented for the free vibration analysis of a rectangular plate with arbitrary edge constraints. In the presented method, natural frequencies and their mode shapes of the plate are calculated by solving an eigenvalue problem of a multi-degree-of-freedom system matrix equation derived by using Lagrange's equations of motion. Characteristic orthogonal polynomials having the property of Timoshenko beam functions which satisfies edge constraints corresponding to those of the objective plate are used. In order to examine the accuracy of the proposed method, numerical examples of the rectangular plates with various thicknesses and edge constraints have been presented. The results have shown good agreement with those of other methods such as an analytic solution, an approximate solution, and a finite element analysis.
\end{abstract}

KEY WORDS: Free vibration analysis; Rectangular plate; Assumed mode method; Characteristic orthogonal polynomials; Lagrange's equation of motion; Arbitrary edge constraints.

\section{INTRODUCTION}

The vibrational characteristic of a large built-up structure such as ships and offshore platforms is crucial design factor in terms of crew's comfort and structural safety. Therefore, in the design stage, free and forced vibration analyses are carried out and the appropriate countermeasures are applied. Because a rectangular plate is extensively used as a structural member of ships and offshore platforms, a number of free vibration analyses are necessarily required for anti-resonance design of plates with respect to the excitation frequencies of main propulsions and auxiliary machineries. In general, these analyses are carried out in the assumption that the plates have classical edge constraints (e.g. simple, free and/or clamped). Most of the plates, however, have different edge constraints from the classical ones by combining with adjacent structural members. Therefore, developing a method which enables to consider the effect of arbitrary edge constraints is necessary.

There exist various methods for the free vibration analysis of a rectangular plate. Mindlin, Schacknow and Deresiewicz (1956) have provided analytic solutions of relatively thick rectangular plates to the edge length. Leissa (1969) has presented analytic solutions of natural frequencies of the various types of plate and the corresponding mode shapes based on trigonometric functions. While these analytic methods may be easily applied in preliminary design stage because of its simplicity, the method is restricted to problems having classical edge constraints such as simple, free and/or clamped edge conditions. In order to

Corresponding author: Dae-Seung Cho

e-mail:daecho@pusan.ac.kr 
overcome this restriction, a number of approximate methods based on an assumed mode method have been suggested, which solve problems having not only classical but also non-classical edge constraints. Dawe and Roufaeil (1980) also have predicted natural frequencies of the square plate having general edge constraints. Gorman (1990) has carried out the free vibration analysis of rectangular plates resting on elastic edge supports by introducing so called Lévy-type solution for lateral displacement, where all the edges are supported simultaneously with same stiffness. Chung, Chung and Kim (1992, 1993) have presented a method using orthogonal polynomials having Timoshenko beam function property, where the edges of the plate are fixed in translational direction but elastically supported with arbitrary stiffness in rotational direction, and Cho, Kim and Jung (1998) have carried out a structural intensity analysis of thin rectangular plate having classical edge boundary conditions. Liu and Liew (1999) have analyzed the vibration of thick rectangular plates with mixed boundary constraints using differential quadrature element method, where the edge of the plate may be partially supported. Zhou (2001) also has analyzed natural frequencies and mode shapes of thick plates with arbitrary edge boundary conditions in both of the translational and rotational direction, where static deflection shape functions of the thick plate have been used as orthogonal polynomials. Lee (2003) has carried out free vibration analyses of orthotropic thick plates using Mindlin plate characteristic functions calculated with the iterative Kantorovich method. $\mathrm{Li}$ (2004) has carried out vibration analyses of rectangular plates with general elastic boundary supports, where the admissible functions composed of a trigonometric function and an arbitrary continuous function have been introduced to ensure the sufficient smoothness of the so-called residual displacement function at the edges. Zhang and Li (2010) have analyzed sound radiation from a baffled rectangular plate with each of its edges arbitrarily supported in the form of elastic restraints where out-of-plane displacements of the plate have been calculated using standard 2-D Fourier cosine series.

In this study, an approximate method based on an assumed mode method is presented to calculate natural frequencies and mode shapes of a rectangular plate having non-classical edge constraints for the translational and rotational direction. The presented method solves an eigenvalue problem of a multi-degree-of-freedom system matrix equation which is derived by using Lagrange's equations of motion. The characteristic orthogonal polynomials with the property of Timoshenko beam functions satisfying the specified edge constraints are used. In order to verify the presented method, numerical examples of the models with various thicknesses and elastic edge constraints are given. The results are compared to those obtained by analytic method, other approximate method, and finite element analysis method.

\section{MATHEMATICAL FORMULATIONS}

\section{Equation of motion and energy}

Mindlin plate theory (Mindlin, Schacknow and Deresiewicz, 1956) is introduced, which includes the effect of shear deformation and rotational inertia, as well, so that it may be applied to not only thin plates but also thick ones of which the thickness to length ratio is larger than one twentieth of half a wavelength.

In the coordinate system as shown in Fig. 1, the equation of motion for an isotropic rectangular plate is as follows;

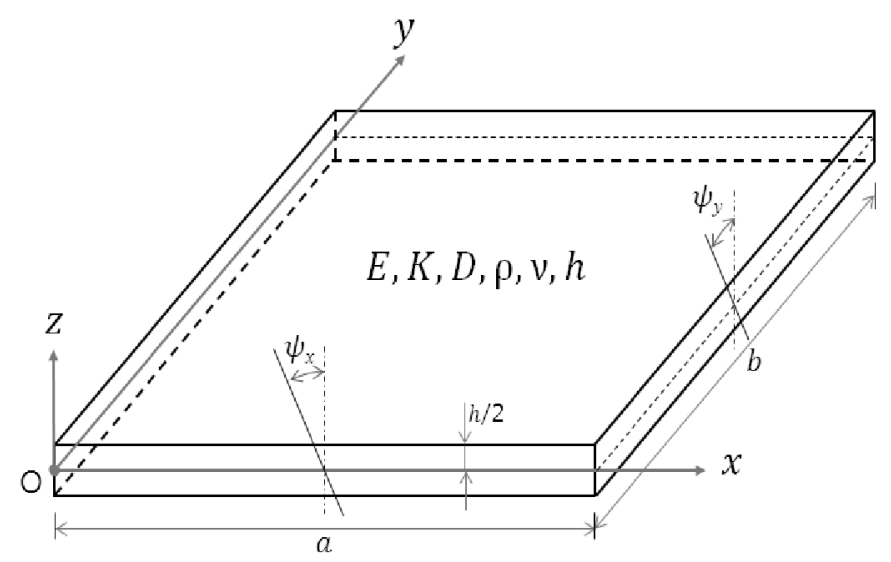

Fig. 1 Coordinates and schematic of rectangular plate. 


$$
\begin{gathered}
\frac{\rho h^{3}}{12} \frac{\partial^{2} \psi_{x}}{\partial t^{2}}-D\left(\frac{\partial^{2} \psi_{x}}{\partial x^{2}}+\frac{1-v}{2} \frac{\partial^{2} \psi_{x}}{\partial y^{2}}+\frac{1+v}{2} \frac{\partial^{2} \psi_{x}}{\partial x \partial y}\right)-K G h\left(\frac{\partial w}{\partial x}-\psi_{x}\right)=0 \\
\frac{\rho h^{3}}{12} \frac{\partial^{2} \psi_{y}}{\partial t^{2}}-D\left(\frac{\partial^{2} \psi_{y}}{\partial y^{2}}+\frac{1-v}{2} \frac{\partial^{2} \psi_{y}}{\partial x^{2}}+\frac{1+v}{2} \frac{\partial^{2} \psi_{y}}{\partial x \partial y}\right)-K G h\left(\frac{\partial w}{\partial y}-\psi_{y}\right)=0 \\
\rho h \frac{\partial^{2} w}{\partial t^{2}}-K G h\left(\frac{\partial^{2} w}{\partial x^{2}}+\frac{\partial^{2} w}{\partial y^{2}}-\frac{\partial \psi_{x}}{\partial x}-\frac{\partial \psi_{y}}{\partial y}\right)=0
\end{gathered}
$$

where $\psi_{x}\left(=\psi_{x}(x, y, t)\right)$ and $\psi_{y}\left(=\psi_{y}(x, y, t)\right)$ are the rotation angle with respect to the $y$ - and $x$-axis, respectively. $w(=w(x, y, t))$ is the lateral displacement with respect to the $x y$-plane. $x$ and $y$ are the in-plane coordinates of the plate and $t$ is the time. $\rho, v, G, K$, and $h$ are the density, Poisson's ratio, shear modulus, shear stiffness correction factor, and thickness, respectively. Also, $D\left(=E h^{3} / 12\left(1-v^{2}\right)\right)$ is the flexural rigidity and $E$ is the Young's modulus.

Meanwhile, when introducing non-dimensional parameters $\xi(=x / a), \eta(=y / b), \alpha(=a / b)$ and $S(=K G h / D)$, the strain energy $V(t)$ and the kinetic energy $T(t)$ of the plate with elastic edge constraints are as follows;

$$
\begin{aligned}
& V(t)=\frac{D}{2 \alpha}\left\{\int _ { 0 } ^ { 1 } \int _ { 0 } ^ { 1 } \left[\left(\frac{\partial \psi_{\xi}}{\partial \xi}\right)^{2}+\alpha^{2}\left(\frac{\partial \psi_{\eta}}{\partial \eta}\right)^{2}+2 \nu \alpha \frac{\partial \psi_{\xi}}{\partial \xi} \frac{\partial \psi_{\eta}}{\partial \eta}+\frac{1-\nu}{2}\left(\alpha \frac{\partial \psi_{\xi}}{\partial \eta}+\frac{\partial \psi_{\eta}}{\partial \xi}\right)^{2}\right.\right. \\
& \left.+S\left(\left(\frac{\partial w}{\partial \xi}-a \psi_{\xi}\right)^{2}+\alpha^{2}\left(\frac{\partial w}{\partial \eta}-a \psi_{\eta}\right)^{2}\right)\right] d \xi d \eta \\
& +\int_{0}^{1}\left[K_{R x 1} \psi_{\xi}^{2}(0, \eta, t)+S K_{T x 1} w^{2}(0, \eta, t)\right] d \eta+\alpha^{2} \int_{0}^{1}\left[K_{R y 1} \psi_{\xi}^{2}(\xi, 0, t)+S K_{T y 1} w^{2}(\xi, 0, t)\right] d \xi \\
& \left.+\int_{0}^{1}\left[K_{R x 2} \psi_{\xi}^{2}(1, \eta, t)+S K_{T x 2} w^{2}(1, \eta, t)\right] d \eta+\alpha^{2} \int_{0}^{1}\left[K_{R y 2} \psi_{\xi}^{2}(\xi, 1, t)+S K_{T y 2} w^{2}(\xi, 1, t)\right] d \xi\right\} \\
& T(t)=\frac{\rho a b}{2} \int_{0}^{1} \int_{0}^{1}\left[\frac{h^{3}}{12}\left(\begin{array}{c}
\cdot 2 \\
\psi_{\xi}+\psi_{\eta}
\end{array}\right)+h w^{\cdot 2}\right] d \xi d \eta
\end{aligned}
$$

where superscript $\cdot$ is the partial derivative with respect to $t$. $K_{T x 1}\left(=k_{T x 1} a / K G h\right), K_{T x 2}\left(=k_{T x 2} a / K G h\right)$, $K_{T y 1}\left(=k_{T y 1} b / K G h\right)$, and $K_{T y 2}\left(=k_{T y 2} b / K G h\right)$ are the non-dimensional stiffnesses at $x=0, x=a, y=0$, and $y=b$, respectively, which correspond to the translational spring constants per unit length $k_{T x 1}, k_{T x 2}, k_{T y 1}$, and $k_{T y 2}$ shown in Fig. 2. In the same manner, $K_{R x 1}\left(=k_{R x 1} a / D\right), K_{R x 2}\left(=k_{R x 2} a / D\right), K_{R y 1}\left(=k_{R y 1} b / D\right)$, and $K_{R y 2}\left(=k_{R y 2} b / D\right)$ are for the rotational spring constants per unit length $k_{R x 1}, k_{R x 2}, k_{R y 1}, k_{R y 2}$ shown in Fig. 2, respectively.

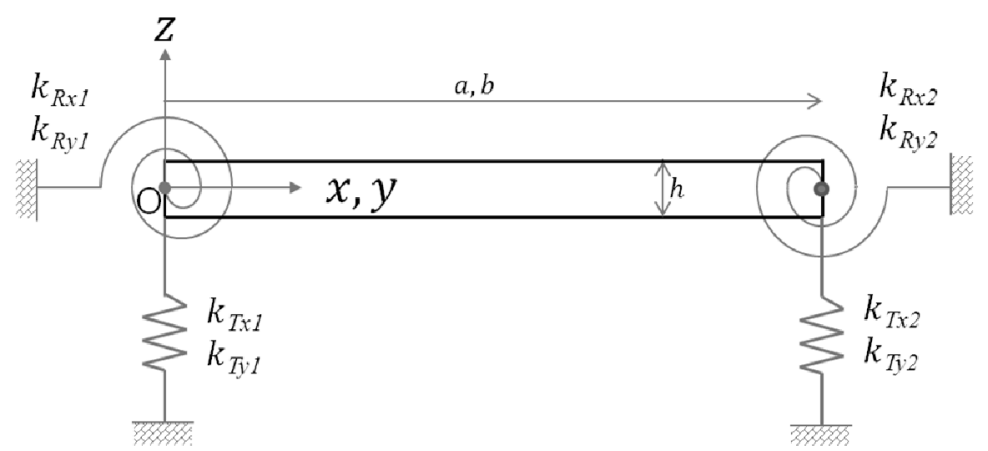

Fig. 2 Rectangular plate elastic-constrained in translational and rotational directions. 


\section{Assumed mode method}

In the assumed mode method, the lateral displacement and the rotation angles are approximated by superposing products of orthogonal polynomials, as follows;

$$
\begin{gathered}
w(\xi, \eta, t)=\sum_{m=1}^{M} \sum_{n=1}^{N} a_{m n}(t) X_{m}(\xi) Y_{n}(\eta) \\
\psi_{\xi}(\xi, \eta, t)=\sum_{m=1}^{M} \sum_{n=1}^{N} b_{m n}(t) \Theta_{m}(\xi) Y_{n}(\eta) \\
\psi_{\eta}(\xi, \eta, t)=\sum_{m=1}^{M} \sum_{n=1}^{N} c_{m n}(t) X_{m}(\xi) \Phi_{n}(\eta)
\end{gathered}
$$

where $X_{m}(\xi), Y_{n}(\eta), \Theta_{m}(\xi)$ and $\Phi_{n}(\eta)$ are the orthogonal polynomials satisfying the specified elastic edge constraints with respect to $\xi$ and $\eta \cdot a_{m n}(t), b_{m n}(t)$ and $c_{m n}(t)$ are the influence coefficients of orthogonal polynomials. Also, $M$ and $N$ are the number of orthogonal polynomials used for approximate solution in $\xi$-and $\eta$-direction, respectively.

Moreover, the equation (4) can be alternatively represented by the following matrix equation.

$$
\{z(\xi, \eta, t)\}=[D(\xi, \eta)]\{q(t)\}
$$

where

$$
\begin{gathered}
\{z(\xi, \eta, t)\}=\left\{w(\xi, \eta, t), \psi \xi(\xi, \eta, t), \psi_{\eta}(\xi, \eta, t)\right\}^{T} \\
{[D(\xi, \eta)]=\left[\begin{array}{rrrrrrrrr}
X_{1} Y_{1} & \cdots & X_{M} Y_{N} & 0 & \cdots & 0 & 0 & \cdots & 0 \\
0 & \cdots & 0 & \Theta_{1} Y_{1} & \cdots & \Theta_{M} Y_{N} & 0 & \cdots & 0 \\
0 & \cdots & 0 & 0 & \cdots & 0 & X_{1} \Phi_{1} & \cdots & X_{M} \Phi_{N}
\end{array}\right]} \\
\{q(t)\}=\left\{\begin{array}{lllllllll}
a_{11} & \cdots & a_{M N} & b_{11} & \cdots & b_{M N} & c_{11} & \cdots & c_{M N}
\end{array}\right\}^{T}
\end{gathered}
$$

Meanwhile, by substituting the equation (2) and (3) into Lagrange's equation of motion below,

$$
\frac{d}{d t}\left(\frac{\partial T(t)}{\partial q_{i}}\right)-\frac{\partial T(t)}{\partial q_{i}}+\frac{\partial V(t)}{\partial q_{i}}=0
$$

the discrete matrix equation with $3 \times M \times N$ degree-of-freedom can be obtained as the following equation.

$$
[M]\{q(t)\}+[K]\{q(t)\}=0
$$

where $[M]$ and $[K]$ are the mass and the stiffness matrix respectively, which are as in APPENDIX.

In the assumption of harmonic motions, i.e.

$$
\{q(t)\}=\{Q\} e^{j \omega t}
$$




$$
\begin{aligned}
& w(\xi, \eta, t)=W(\xi, \eta) e^{j \omega t} \\
& \psi_{\xi}(\xi, \eta, t)=\Psi_{\xi}(\xi, \eta) e^{j \omega t} \\
& \psi_{\eta}(\xi, \eta, t)=\Psi_{\eta}(\xi, \eta) e^{j \omega t}
\end{aligned}
$$

the equation (7) leads an eigenvalue problem which gives natural frequencies and eigenvectors of the system, where $j$ is the unit imaginary, $\omega$ is the angular frequency.

Finally, the mode shape corresponding to each natural frequency is obtained from the following equation.

$$
\left\{W(\xi, \eta), \Psi_{\xi}(\xi, \eta), \Psi_{\eta}(\xi, \eta)\right\}_{l}^{T}=[D(\xi, \eta)]\{Q\}_{l}
$$

where $l$ is the order of mode.

\section{Characteristic orthogonal polynomials}

When using the assumed mode method, the orthogonal polynomials suitable to the property of the target model should be applied for accurate analysis. For this purpose, the characteristic orthogonal polynomials having the property of Timoshenko beam functions which satisfies the specified edge constraints have been derived.

The lateral motion of Timoshenko beam which corresponds to a unit width strip taken from the particular Mindlin rectangular plate under consideration in the direction parallel to the edge of the plate follows the equation (10).

$$
\begin{gathered}
\rho I \frac{\partial^{2} \psi(x, t)}{\partial t^{2}}-E I \frac{\partial^{2} \psi(x, t)}{\partial x^{2}}-K A G\left(\frac{\partial w(x, t)}{\partial x}-\psi(x, t)\right)=0 \\
\rho A \frac{\partial^{2} w(x, t)}{\partial t^{2}}-K A G\left(\frac{\partial w^{2}(x, t)}{\partial x^{2}}-\frac{\partial \psi(x, t)}{\partial x}\right)=0
\end{gathered}
$$

where $w(x, t)$ and $\psi(x, t)$ are the lateral deflection and the rotational angle of the beam, respectively. $A$ and $I$ are the cross sectional area and the second moment of inertia of the beam section, respectively.

When assuming the harmonic motion, i.e.

$$
w(x, t)=W(x) e^{j \omega t}, \psi(x, t)=\Psi(x) e^{j \omega t}
$$

the equation (10) can be rewritten with a non-dimensional parameter $\zeta=x / L$ as shown in the equation (12).

$$
\begin{gathered}
\rho I \omega^{2} \Psi+K A G\left(\frac{W^{\prime}}{L}-\Psi\right)+E I \frac{W^{\prime \prime}}{L^{2}}=0 \\
\rho A \omega^{2} W+K A G\left(\frac{W^{\prime \prime}-L \Psi^{\prime}}{L^{2}}\right)=0
\end{gathered}
$$

where $L$ is the length of the beam. Also, superscript ' is the partial derivative with respect to $\zeta \cdot W(\zeta)$ and $\Psi_{1}(\zeta)$ are the 
Timoshenko beam functions, which should satisfy the following edge constraints.

$$
\begin{gathered}
W(0)=-\frac{W^{\prime}(0)-L \Psi(0)}{K_{T 1}}, \quad \Psi(0)=\frac{\Psi^{\prime}(0)}{K_{R 1}} \\
W(1)=\frac{W^{\prime}(1)-L \Psi(1)}{K_{T 2}}, \quad \Psi(1)=-\frac{\Psi^{\prime}(1)}{K_{R 2}}
\end{gathered}
$$

where $K_{T 1}\left(=k_{T 1} L / K G A\right), K_{R 1}\left(=k_{R 1} L / E I\right), K_{T 2}\left(=k_{T 2} L / K G A\right)$, and $K_{R 2}\left(=k_{R 2} L / E I\right) . k_{T 1}, k_{R 1}, k_{T 2}$, and $k_{R 2}$ are the spring constants with respect to the translation and the rotation at two edges, respectively.

After eliminating $\omega^{2}$ from the equation (12), by introducing non-dimensional parameters $r^{2}=I / A L^{2}$ and $s^{2}=E I / K A G L^{2}$, the equation (12) becomes the following equation.

$$
-r^{2}\left(W^{\prime \prime}-L \Psi^{\prime}\right) \Psi+\left(\frac{W^{\prime}}{L}-\Psi+s^{2} \Psi^{\prime \prime}\right) W=0
$$

For thick beams, it is general that shear deflection effects are remarkably larger than rotational inertia one so that $r^{2} \approx 0$ (Chung, Chung and Kim, 1993). Therefore, the equation (14) can be simplified to the following equation.

$$
W^{\prime} \approx L\left(\Psi-s^{2} \Psi^{\prime \prime}\right)
$$

Moreover, Timoshenko beam functions should satisfy the following orthogonality.

$$
\int_{0}^{1}\left(\rho A W_{m} W_{n}+\rho I \Psi_{m} \Psi_{n}\right) d \zeta= \begin{cases}0, & m \neq n \\ 1, & m=n\end{cases}
$$

where $W_{m}$ and $\Psi_{m}$ are the $m$-th order Timoshenko beam functions.

Meanwhile, when setting the first-order Timoshenko beam function of rotation $\Psi_{1}(\zeta)$ to the equation (17), that of translation $W_{1}(\zeta)$ is derived as the equation (18).

$$
\begin{gathered}
\Psi_{1}(\zeta)=A_{1}\left(A_{31} \zeta^{3}+A_{21} \zeta^{2}+A_{11} \zeta+1\right) \\
W_{2}(\zeta)=A_{2} L\left[\frac{A_{31}}{4} \zeta^{4}+\frac{A_{21}}{3} \zeta^{3}+\frac{A_{11}-6 A_{31} s^{2}}{2} \zeta^{2}+\left(1-2 A_{21} s^{2}\right) \zeta+C\right]
\end{gathered}
$$

where $A_{1}$ is the coefficient which satisfy the equation (16) when $m=n=1$, and the rest coefficients are obtained as the equation (19) by using boundary conditions of the equation (13).

$$
A_{11}=K_{R 1}, \quad A_{21}=\frac{K_{T 1}}{2 s^{2}} C, \quad A_{31}=-\hat{K}_{1}-\hat{K}_{2} \frac{K_{T 1}}{2 s^{2}} C, C=\hat{P} / \hat{Q}
$$




$$
\begin{gathered}
\hat{K}_{1}=\frac{K_{R 1}+K_{R 1} K_{R 2}+K_{R 2}}{K_{R 2}+3}, \quad \hat{K}_{2}=\frac{K_{R 2}+2}{K_{R 2}+3}, \\
\hat{P}=\hat{K}_{1}\left(\frac{K_{T 2}}{4}-3 s^{2} K_{T 2}+6 s^{2}\right)-K_{T 2}\left(\frac{K_{R 1}}{2}+1\right), \\
\hat{Q}=-\left(\frac{K_{T 2}}{4}-3 s^{2} K_{T 2}+6 s^{2}\right) \frac{\hat{K}_{2} K_{T 1}}{2 s^{2}}+\left(\frac{K_{T 2}}{3}-2 s^{2} K_{T 2}+2 s^{2}\right) \frac{K_{T 1}}{2 s^{2}}+K_{T 2}
\end{gathered}
$$

In the same manner, when setting the second-order Timoshenko beam function of rotation $\Psi_{2}(\zeta)$ to the equation (20), that of translation $W_{2}(\zeta)$ is derived as the equation $(21)$.

$$
\begin{gathered}
\Psi_{2}(\zeta)=A_{2}\left(\zeta^{4}+A_{32} \zeta^{3}+A_{22} \zeta^{2}+A_{12} \zeta+A_{02}\right) \\
W_{2}(\zeta)=A_{2} L\left[\frac{1}{5} \zeta^{5}+\frac{A_{32}}{4} \zeta^{4}+\left(\frac{A_{22}}{3}-4 s^{2}\right) \zeta^{3}+\left(\frac{A_{12}}{2}-3 A_{32} s^{2}\right) \zeta^{2}+\left(A_{02}-2 A_{22} s^{2}\right) \zeta+D\right]
\end{gathered}
$$

where $A_{2}$ also is the coefficient which satisfies the equation (16) when $m=n=2$, and the rest coefficients are obtained as the equation (22) by using the equation (13)

$$
\begin{aligned}
& A_{32}=-\bar{D} / \bar{C}, A_{22}=A_{32} \bar{A}+\bar{B}, \quad A_{12}=-\left(\bar{T} A_{22}+\bar{S} A_{32}+\bar{R}\right), \\
& A_{02}=A_{12} / K_{R 1}, \quad D=2 s^{2} A_{22} / K_{T 1}, \quad \bar{T}=\frac{K_{R 1}\left(K_{R 2}+2\right)}{K_{R 1}+K_{R 1} K_{R 2}+K_{R 2}}, \\
& \bar{S}=\frac{K_{R 1}\left(K_{R 2}+3\right)}{K_{R 1}+K_{R 1} K_{R 2}+K_{R 2}}, \quad \bar{R}=\frac{K_{R 1}\left(K_{R 2}+4\right)}{K_{R 1}+K_{R 1} K_{R 2}+K_{R 2}}, \\
& \bar{A}=\frac{\frac{1}{4}-3 s^{2}+\frac{6 s^{2}}{K_{T 2}}-\bar{S}\left(\frac{1}{2}+\frac{1}{K_{R 1}}\right)}{-\frac{1}{T}\left(\frac{1}{2}+\frac{1}{K_{R 1}}\right)+2 s^{2}\left(1-\frac{1}{K_{T 2}}-\frac{1}{K_{T 1}}\right)-\frac{1}{3}}, \quad \bar{B}=\frac{\frac{1}{5}-4 s^{2}+\frac{12 s^{2}}{K_{T 2}}-\frac{-}{R}\left(\frac{1}{2}+\frac{1}{K_{R 1}}\right)}{-\left(\frac{1}{2}+\frac{1}{K_{R 1}}\right)+2 s^{2}\left(1-\frac{1}{K_{T 2}}-\frac{1}{K_{T 1}}\right)-\frac{1}{3}}, \\
& \bar{C}=\int_{0}^{1}\left\{\rho A L\left[\frac{1}{4} \zeta^{4}+\frac{\bar{A}}{3} \zeta^{3}-\left(\frac{\bar{T} \bar{A}+\bar{S}}{2}+3 s^{2}\right) \zeta^{2}-\left(\frac{\bar{T} \bar{A}+\bar{S}}{K_{R 1}}+2 \bar{A} s^{2}\right) \zeta+\frac{2 \bar{A} s^{2}}{K_{T 1}}\right] W_{1}\right. \\
& \left.+\rho I\left[\zeta^{3}+\bar{A} \zeta^{2}-(\bar{T} \bar{A}+\bar{S}) \zeta-\frac{\bar{T} \bar{A}+\bar{S}}{K_{R 1}}\right] \Psi_{1}\right\} d \zeta
\end{aligned}
$$




$$
\begin{aligned}
\bar{D} & =\int_{0}^{1}\left\{\rho A L\left[\frac{1}{5} \zeta^{5}+\left(\frac{\bar{B}}{3}-4 s^{2}\right) \zeta^{3}-\left(\frac{\bar{T} \bar{B}+\bar{R}}{2}\right) \zeta^{2}-\left(\frac{\bar{T} \bar{B}+\bar{R}}{K_{R 1}}+2 \bar{B} s^{2}\right) \zeta+\frac{2 \bar{B} s^{2}}{K_{T 1}}\right] W_{1}\right. \\
& \left.+\rho I\left[\zeta^{4}+\bar{B} \zeta^{2}-(\bar{T} \bar{B}+\bar{R}) \zeta-\frac{\bar{T} \bar{B}+\bar{R}}{K_{R 1}}\right] \Psi_{1}\right\} d \zeta
\end{aligned}
$$

Finally, the $k$-th order Timoshenko beam function can be successively obtained by using the orthogonal polynomial property of the equation (23) and (24).

$$
\begin{gathered}
W_{k}=A_{k}\left[\left(\zeta-B_{k}\right) W_{k-1}-C_{k} W_{k-2}\right] \\
\Psi_{k}=A_{k}\left[\left(\zeta-B_{k}\right) \Psi_{k-1}-C_{k} \Psi_{k-2}+\frac{W_{k-1}}{L}\right]
\end{gathered}
$$

where $A_{k}$ is the coefficient which satisfies the equation (16) when $m=n=k . \quad B_{k}$ and $C_{k}$ are as follows;

$$
\begin{gathered}
B_{k}=\int_{0}^{1}\left[\zeta\left(\rho A W_{k-1}^{2}+\rho I \Psi_{k-1}^{2}\right)\right] d \zeta, \\
C_{k}=\int_{0}^{1}\left[\zeta\left(\rho A W_{k-1} W_{k-2}+\rho I \Psi_{k-1} \Psi_{k-2}\right)\right] d \zeta
\end{gathered}
$$

In practice, the same type of polynomials derived here, $W_{k}$ and $\Psi_{k}$, is used for $\xi$ and $\eta$ directions of the objective plate.

\section{NUMERICAL ANALYSIS}

In order to verify the accuracy of the presented method, numerical examples of a square and a rectangular plate have been presented. The results have been compared to those of analytic, approximate, and finite element solutions. The Young's modulus, density, and Poisson's ratio of the plates are set to $2.1 \times 10^{11} \mathrm{~N} / \mathrm{m}^{2}, 7850 \mathrm{~kg} / \mathrm{m}^{3}$ and 0.3 , respectively. The number of polynomials has been set to 10 for $\xi$ and $\eta$ directions of the objective models after convergence test.

\section{Square plate}

Free vibration analyses of the square plate with classical edge constraints have been carried out. The size of the square plate is $1.0 \mathrm{~m} \times 1.0 \mathrm{~m}$ and the thickness is $0.1 \mathrm{~m}$.

Table 1 shows the lowest 6 natural frequencies for the square plate of which all 4 edges are simply supported (simplesimple-simple-simple, SSSS). The results of the presented method are coincident with those of analytic solution by Mindlin, Schacknow and Deresiewicz (1956, hereafter 'Mindlin's'). Table 2 compares the natural frequencies of the square plate by the presented method with those by approximate solutions of Dawe and Roufaeil (1980, hereafter 'Dawe's'), of which 2 edges facing each other are simply supported but the other 2 edges clamped (simple-clamped-simple-clamped, SCSC). The results show maximally $0.3 \%$ difference comparing with those by approximate solution. Finally, Table 3 shows the results of the square plate of which all 4 edges are clamped (clamped-clamped-clamped-clamped, CCCC). When comparing with those by 
the Dawe's approximate solution, the presented method provides good accuracy with maximally $0.5 \%$ difference. For information, originally, the Mindlin's and Dawe's had been indicated as non-dimensional natural frequency, but the data in the Tables have been converted to corresponding natural frequencies.

Table 1 Natural frequencies in $H z$ of $1 m \times 1 m$ square plate with thickness of $0.1 m$ (SSSS).

\begin{tabular}{|c|c|c|c|c|}
\hline Order & Mode & Mindlin's & Present & Difference $(\%)$ \\
\hline 1 & $(1,1)$ & 474.8 & 474.8 & 0.0 \\
\hline 2 & $(2,1)$ & $1,132.3$ & $1,132.2$ & 0.0 \\
\hline 3 & $(1,2)$ & $1,132.3$ & $1,132.2$ & 0.0 \\
\hline 4 & $(2,2)$ & $1,736.8$ & $1,736.7$ & 0.0 \\
\hline 5 & $(3,1)$ & $2,115.6$ & $2,115.6$ & 0.0 \\
\hline 6 & $(1,3)$ & $2,115.6$ & $2,115.6$ & 0.0 \\
\hline
\end{tabular}

Table 2 Natural frequencies in $H z$ of $1 m \times 1 m$ square plate with thickness of $0.1 m$ (SCSC).

\begin{tabular}{|c|c|c|c|c|}
\hline Order & Mode & Dawe's & Present & Difference $(\%)$ \\
\hline 1 & $(1,1)$ & 664.7 & 663.8 & -0.1 \\
\hline 2 & $(2,1)$ & $1,224.2$ & $1,222.3$ & -0.2 \\
\hline 3 & $(1,2)$ & $1,474.4$ & $1,472.9$ & -0.1 \\
\hline 4 & $(2,2)$ & $1,966.5$ & $1,960.2$ & -0.3 \\
\hline 5 & $(3,1)$ & $2,163.1$ & $2,160.3$ & -0.1 \\
\hline 6 & $(1,3)$ & $2,521.4$ & $2,519.8$ & -0.1 \\
\hline
\end{tabular}

Table 3 Natural frequencies in $H z$ of $1 m \times 1 m$ square plate with thickness of $0.1 m$ (CCCC).

\begin{tabular}{|c|c|c|c|c|}
\hline Order & Mode & Dawe's & Present & Difference $(\%)$ \\
\hline 1 & $(1,1)$ & 813.8 & 812.3 & -0.2 \\
\hline 2 & $(2,1)$ & 1555.0 & $1,551.7$ & -0.2 \\
\hline 3 & $(1,2)$ & 1555.0 & $1,551.7$ & -0.2 \\
\hline 4 & $(2,2)$ & 2187.6 & $2,176.4$ & -0.5 \\
\hline 5 & $(3,1)$ & 2570.4 & $2,565.4$ & -0.2 \\
\hline 6 & $(1,3)$ & 2592.4 & $2,589.6$ & -0.1 \\
\hline
\end{tabular}

\section{Rectangular plate}

Numerical analyses of a rectangular plate having non-classical edge constraints have been carried out, of which the size is 4 $m \times 3 m$ and the 3 edges except the edge at $y=3 m$ are simply supported. In order to verify the accuracy of the presented method, the results have been compared with finite element solution by MSC/NASTRAN (MSC, 2010), in which CQUAD4 has been used to build up the objective model.

Fig. 3 shows the results of the plate of which the edge at $y=3 \mathrm{~m}$ is free (SSFS) and the thicknesses are $0.01,0.05$ and $0.1 \mathrm{~m}$. The results are good agreed with those of finite element method and show that the natural frequencies grow as the thickness increases. Moreover, Fig. 4 presents the first-, third-, and forth-order mode shape representatively, of which the thickness is $0.05 \mathrm{~m}$. The results show good agreement with finite element solution. 


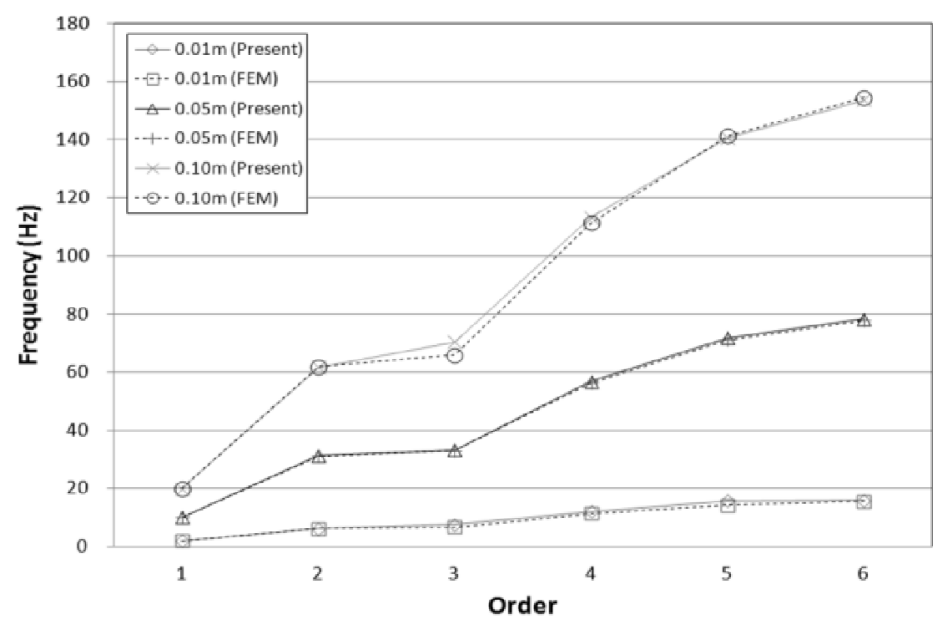

Fig. 3 Natural frequencies in $\mathrm{Hz}$ of $4 \mathrm{~m} \times 3 \mathrm{~m}$ rectangular plate with various thicknesses (SSFS).

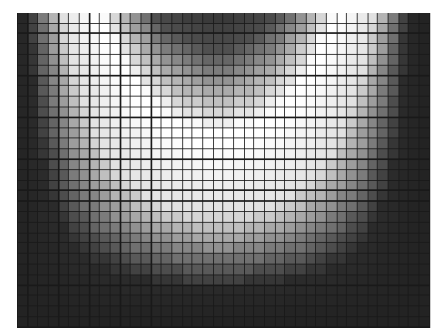

Present (N.F: $10.1 \mathrm{~Hz}$ )

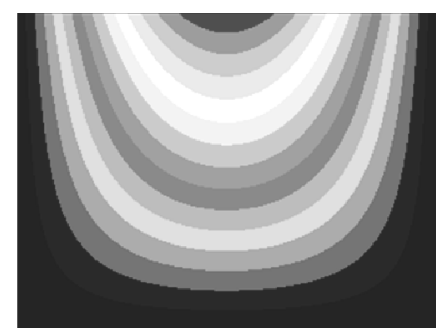

FEM (N.F: $10.1 \mathrm{~Hz}$ )

(a) $1^{\text {st }}$ Mode shape.

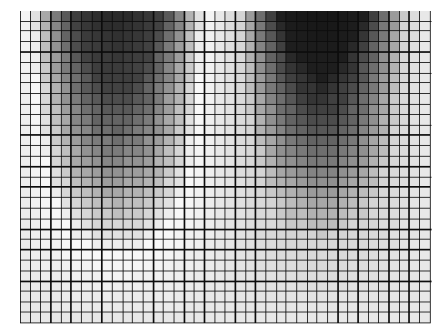

Present (N.F: $33.1 \mathrm{~Hz}$ )

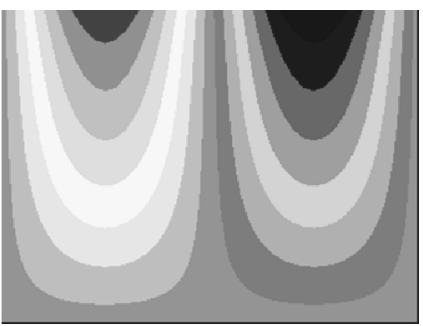

FEM (N.F: $33.2 \mathrm{~Hz}$ )

(b) $3^{\text {rd }}$ Mode shape.

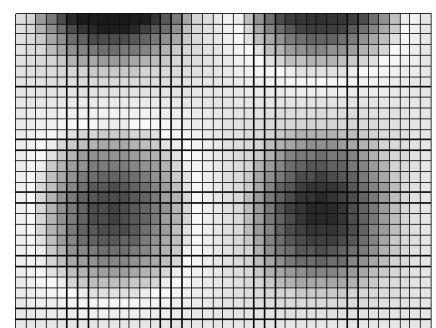

Present (N.F: $56.8 \mathrm{~Hz}$ )

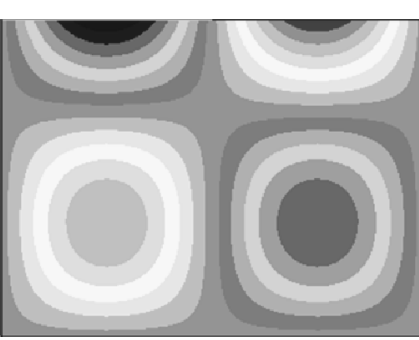

FEM (N.F: $56.4 \mathrm{~Hz}$ )

(c) $4^{\text {th }}$ Mode shape.

Fig. 4 Mode shape of $4 m \times 3 m$ rectangular plate with thickness of $0.05 m$ (SSFS, N.F; natural frequency).

Meanwhile, Fig. 5 and Fig. 6 show the results of the rectangular plate of which the thickness is $0.1 \mathrm{~m}$ and the edge on $y=3$ $m$ is elastically supported in the direction of translation and rotation, respectively. The stiffness per unit length of the spring varies from $10^{5}$ to $10^{10} \mathrm{~N} / \mathrm{m}^{2}$ (or $\mathrm{N} / \mathrm{rad} \cdot \mathrm{m}$ ). The results also show good agreement with finite element solutions. The natural frequencies also gradually grow up to the specific frequency as the stiffness increases. 


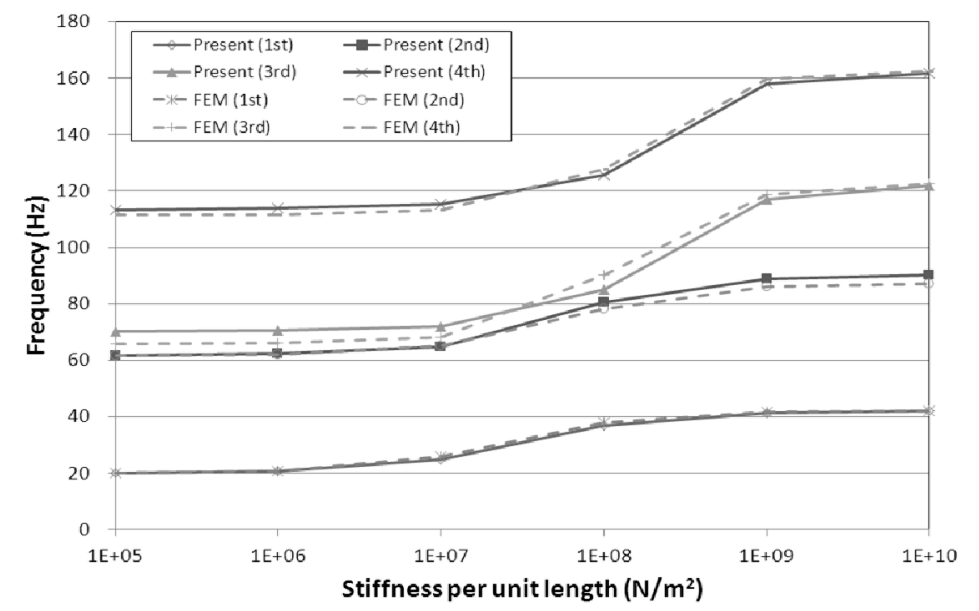

Fig. 5 Natural frequencies of $4 m \times 3 m$ rectangular plate with thickness of $0.1 m$ (elastically 1-edge restrained in translation).

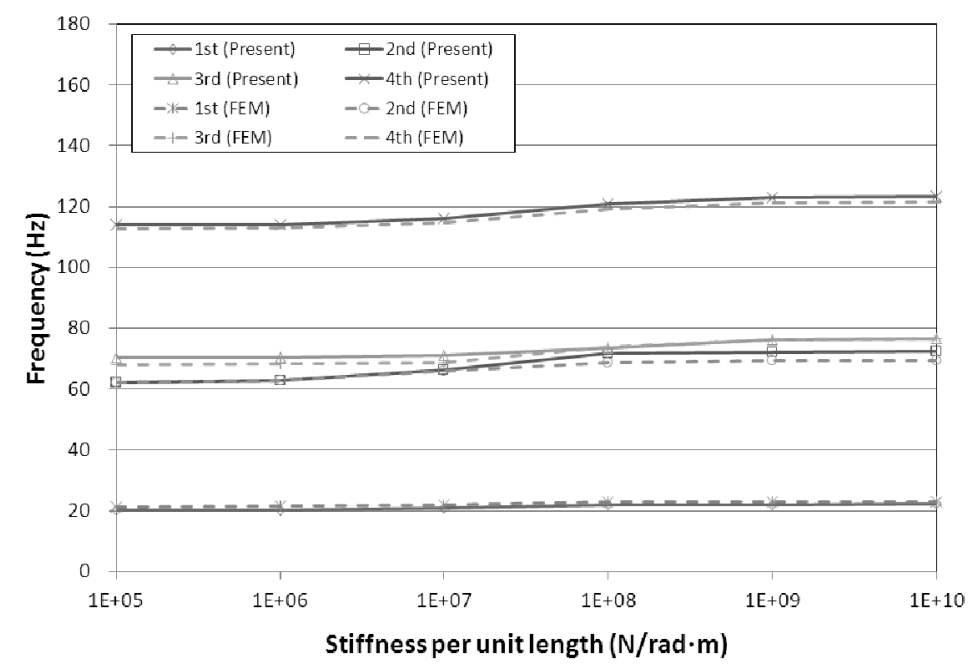

Fig. 6 Natural frequencies of $4 m \times 3 m$ rectangular plate with thickness of $0.1 \mathrm{~m}$ (elastically 1-edge restrained in rotation).

\section{CONCLUSIONS}

A numerical method has been presented for free vibration analyses of rectangular plates with non-classical edge constraints for translational and rotational direction. For this purpose, an approximate solution has been adopted based on an assumed mode method. In the presented method, natural frequencies and the mode shapes are calculated by solving an eigenvalue problem of a multi-degree-of-freedom system matrix equation which is derived by using Lagrange's equations of motion. And the characteristic orthogonal polynomials having the property of Timoshenko beam functions which satisfy the specified edge constraints are adopted. In order to verify the presented method, numerical examples of a square and a rectangular plate model with various thicknesses and edge constraints have been presented. The results have showed good agreement with those of other methods such as an analytic solution, an approximate solution, and a finite element analysis. It is expected that the presented method could be efficiently used for free vibration analysis of rectangular plate having not only classical but also nonclassical edge constraints.

\section{ACKNOWLEDGEMENTS}

This work was supported by the National Research Foundation of Korea (NRF) grant funded by the Korea government (MEST) through GCRC-SOP (Grant No. 2011-0030669 and No. 2011-0030686). 


\section{REFERENCES}

Cho, D.S., Kim, S.S. and Jung, S.M., 1998. Structural intensity analysis of stiffened plate using assumed mode method. Journal of the Society of Naval Architects of Korea, 35(4), pp.76-86.

Chung, J.H., Chung, T.Y. and Kim, K.C., 1992. Vibration analysis of mindlin plates using polynomials having the property of Timoshenko beam functions. Journal of the Society of Naval Architects of Korea, 29(1), pp.158-172.

Chung, J.H., Chung, T.Y. and Kim, K.C, 1993. Vibration analysis of orthotropic Mindlin plates with edges elastically restrained against rotation. Journal of Sound and Vibration, 163(1), pp.151-163.

Dawe, D.J. and Roufaeil, O.L., 1980. Rayleigh-Ritz vibration analysis of Mindlin plates. Journal of Sound and Vibration, 69(3), pp.345-359.

Gorman, D.J., 1990. A general solution for the free vibration of rectangular plates resting on uniform elastic edge supports. Journal of Sound and Vibration, 139(2), pp.325-335.

Lee, J.M., 2003. Vibration analysis of thick orthotropic plates using mindlin plate characteristic functions. Journal of Ocean Engineering and Technology, 17(3), pp.21-26.

Leissa, A.W., 1969. Vibration of plates, NASA Report No SP-160. Scientific and Technical Information Division, National Aeronautics and Space Administration.

Li, W.L., 2004. Vibration analysis of rectangular plates with general elastic boundary supports. Journal of Sound and Vibration, 273(3), pp.619-635.

Liu, F.-L. and Liew, K.M., 1999. Analysis of vibrating thick rectangular plates with mixed boundary constraints using differential quadrature element method. Journal of Sound and Vibration, 225(5), pp.915-934.

Mindlin, R.D., Schacknow, A. and Deresiewicz, H., 1956. Flexural vibrations of rectangular plates. Journal of Applied Mechanics, 23, pp.430-436.

MSC, 2010. MD Nastran 2010 Dynamic analysis user's guide. MSC Software.

Zhang, X. and Li, W.L., 2010. A unified approach for predicting sound radiation from baffled rectangular plates with arbitrary boundary conditions. Journal of Sound and Vibration, 329(25), pp.5307-5320.

Zhou, D., 2001. Vibrations of Mindlin rectangular plates with elastically restrained edges using static Timoshenko beam functions with the Rayleigh-Ritz method. International Journal of Solids and Structures, 38(32-33), pp.5565-5580.

\section{APPENDIX: Mass and stiffness matrix}

$[M]$ and $[K]$ are the mass and stiffness matrix, respectively, of which the dimension is $(3 \times M \times N)$ by $(3 \times M \times N)$, and the elements $m_{p q}$ and $k_{p q}$ are calculated as follows;

\section{Mass matrix element, $m_{p q}$}

- $p=(i-1) N+j, q=(m-1) N+n$

$$
m_{p q}=\rho a b h \int_{0}^{1} X_{m} X_{i} d \xi \int_{0}^{1} Y_{n} Y_{j} d \eta
$$

- $p=(i+M-1) N+j, q=(m+M-1) N+n$

$$
m_{p q}=\frac{\rho a b h^{3}}{12} \int_{0}^{1} \Theta_{m} \Theta_{i} d \xi \int_{0}^{1} Y_{n} Y_{j} d \eta
$$

- $p=(i+M-1) N+j, q=(m+M-1) N+n$

$$
m_{p q}=\frac{\rho a b h^{3}}{12} \int_{0}^{1} X_{m} X_{i} d \xi \int_{0}^{1} \Phi_{n} \Phi_{j} d \eta
$$


- others

$$
m_{p q}=0
$$

where $i, m=1,2, \cdots, M$ and $j, n=1,2, \cdots, N$.

Stiffness matrix element, $k_{p q}$

- $p=(i-1) N+j$ and $q=(m-1) N+n$

$$
\begin{array}{r}
k_{p q}=\frac{1}{\alpha}\left[K h G\left(\int_{0}^{1} X_{m}^{\prime} X_{i}^{\prime} d \xi \int_{0}^{1} Y_{n} Y_{j} d \eta\right)+\alpha^{2} K h G\left(\int_{0}^{1} X_{m} X_{i} d \xi \int_{0}^{1} Y_{n}^{\prime} Y_{j}^{\prime} d \eta\right)\right] \\
+\frac{K h G}{\alpha}\left[K_{T x 1} X_{m}(0) X_{i}(0)+K_{T x 2} X_{m}(1) X_{i}(1)\right] \int_{0}^{1} Y_{n} Y_{j} d \eta \\
+\alpha K h G\left[K_{T y 1} Y_{n}(0) Y_{j}(0)+K_{T y 2} Y_{n}(1) Y_{j}(1)\right] \int_{0}^{1} X_{m} X_{i} d \xi
\end{array}
$$

- $p=(i-1) N+j, q=(m+M-1) N+n$

$$
k_{p q}=-\frac{a}{\alpha} K h G \int_{0}^{1} \Theta_{m} X_{i}^{\prime} d \xi \int_{0}^{1} Y_{n} Y_{j} d \eta
$$

- $p=(i-1) N+j, q=(m+2 M-1) N+n$

$$
k_{p q}=-\alpha b K h G \int_{0}^{1} X_{m} X_{i} d \xi \int_{0}^{1} \Phi_{n} Y_{j}^{\prime} d \eta
$$

- $p=(i+M-1) N+j, q=(m-1) N+n$

$$
k_{p q}=-\frac{a}{\alpha} K h G \int_{0}^{1} X_{m}^{\prime} \Theta_{i} d \xi \int_{0}^{1} Y_{n} Y_{j} d \eta
$$

- $p=(i+M-1) N+j, q=(m+M-1) N+n$

$$
\begin{gathered}
k_{p q}=\frac{D}{\alpha} \int_{0}^{1} \Theta_{m}^{\prime} \Theta_{i}^{\prime} d \xi \int_{0}^{1} Y_{n} Y_{j} d \eta+\alpha D \int_{0}^{1} \Theta_{m} \Theta_{i} d \xi \int_{0}^{1} Y_{n}^{\prime} Y_{j}^{\prime} d \eta+\frac{K h G a^{2}}{\alpha} \int_{0}^{1} \Theta_{m} \Theta_{i} d \xi \int_{0}^{1} Y_{n} Y_{j} d \eta \\
+\frac{D}{\alpha}\left[K_{R x 1} \Theta_{m}(0) \Theta_{i}(0)+K_{R x 2} \Theta_{m}(1) \Theta_{i}(1)\right] \int_{0}^{1} Y_{n} Y_{j} d \eta
\end{gathered}
$$

- $p=(i+M-1) N+j, q=(m+2 M-1) N+n$

$$
k_{p q}=D\left(\int_{0}^{1} X_{m}^{\prime} \Theta_{i} d \xi \int_{0}^{1} \Phi_{n} Y_{j}^{\prime} d \eta+v \int_{0}^{1} X_{m} \Theta_{i}^{\prime} d \xi \int_{0}^{1} \Phi_{n}^{\prime} Y_{j} d \eta\right)
$$


- $p=(i+2 M-1) N+j, q=(m-1) N+n$

$$
k_{p q}=-\alpha K h G b \int_{0}^{1} X_{m} X_{i} d \xi \int_{0}^{1} Y_{n}^{\prime} \Phi_{j} d \eta
$$

- $p=(i+2 M-1) N+j, q=(m+M-1) N+n$

$$
k_{p q}=D\left(\int_{0}^{1} \Theta_{m} X_{i}^{\prime} d \xi \int_{0}^{1} Y_{n}^{\prime} \Phi_{j} d \eta+v \int_{0}^{1} \Theta_{m}^{\prime} X_{i} d \xi \int_{0}^{1} Y_{n} \Phi_{j}^{\prime} d \eta\right)
$$

- $p=(i+2 M-1) N+j, q=(m+2 M-1) N+n$

$$
\begin{gathered}
k_{p q}=\alpha D \int_{0}^{1} X_{m} X_{i} d \xi \int_{0}^{1} \Phi_{n}^{\prime} \Phi_{j}^{\prime} d \eta+\frac{D}{\alpha} \int_{0}^{1} X_{m}^{\prime} X_{i}^{\prime} d \xi \int_{0}^{1} \Phi_{n} \Phi_{j} d \eta+\alpha K h G b^{2} \int_{0}^{1} X_{m} X_{i} d \xi \int_{0}^{1} \Phi_{n} \Phi_{j} d \eta \\
+\alpha D\left[K_{R y 1} \Phi_{n}(0) \Phi_{j}(0)+K_{R y 2} \Phi_{n}(1) \Phi_{j}(1)\right] \int_{0}^{1} X_{m} X_{i} d \xi .
\end{gathered}
$$

